RAIRO Operations Research

RAIRO Oper. Res. 37 (2003) 249-271

DOI: $10.1051 /$ ro $: 2004008$

\title{
CONSTRUCTION DE FACETTES POUR LE POLYTOPE DU SAC-À-DOS QUADRATIQUE EN 0-1
}

\author{
Alain Faye $^{1}$ et Olivier Boyer ${ }^{1}$
}

\begin{abstract}
We build facets of the quadratic 0-1 knapsack polytope following two different approaches. The quadratic 0-1 knapsack polytope is included in the Boolean quadric polytope introduced by Padberg [12] for unconstrained 0-1 quadratic problem. So in a first approach, we ask the question which are the facets of the Boolean quadric polytope that are still facets of the quadratic 0-1 knapsack polytope. Results for this problem are given for the cut inequality introduced by Padberg [12]. We give necessary and sufficient conditions for which the cut inequality induces a facet of the quadratic 0-1 knapsack polytope and when these conditions are not satisfied we give a lifting of the inequality. In a different way, following the linearization technique of Adams and Sherali [1], we build facets of the quadratic 0-1 knapsack polytope from facets of the linear 0-1 knapsack polytope multiplying a linear inequality by a variable $x_{i}$ or $\bar{x}_{i}=1-x_{i}$. We show that this approach gives facets of the quadratic 0-1 knapsack polytope and we extend it to multiplying an inequality that induces a facet of the quadratic 0-1 knapsack polytope. To conclude, we give numerical results of a cutting plane algorithm involving cuts built following these two schemes.
\end{abstract}

Résumé. Nous construisons des familles de facettes du polytope du sac-à-dos quadratique en 0-1 selon les deux approches suivantes. Le Boolean quadric polytope (introduit dans le cas sans contraintes par Padberg [12]) contenant le polytope du sac-à-dos quadratique, une première approche consiste à se demander sous quelles conditions une facette du premier est aussi une facette du second et quand ces conditions ne sont pas remplies quels liftings permettent d'en faire une facette. Des réponses à ces questions sont données dans le cas de l'inégalité « coupe » introduite par Padberg. Dans une seconde approche, suivant la méthode de linéarisation d'Adams et Sherali [1], nous multiplions par une variable directe ou complémentée une facette du polytope du sac-à-dos linéaire. Nous montrons que cette approche permet d'obtenir

${ }^{1}$ CEDRIC-IIE, 18 allée Jean Rostand, 91025 Evry Cedex, France; e-mail : fayea@iie.cnam.fr

(c) EDP Sciences 2004 
des facettes du polytope du sac-à-dos quadratique et nous l'étendons par la suite à la multiplication de facettes du sac-à-dos quadratique lui-même. Des résultats numériques illustrent la mise en oeuvre dans un algorithme de coupes, d'inégalités ainsi obtenues.

Mots Clés. Polytope du sac-à-dos quadratique en 0-1, méthodes polyédriques.

\section{INTRODUCTION}

Étant donné un graphe $G=(V, E)$, des poids $a_{i} \geq 0 i \in V, b$ t.q. $0<b<$ $\sum_{i \in V} a_{i}$ et des coûts $c_{i} i \in V, c_{i j} \neq 0(i, j) \in E$, on définit le problème du sac-à-dos quadratique en 0-1 par :

$$
\max \sum_{i \in V} c_{i} x_{i}+\sum_{(i, j) \in E} c_{i j} x_{i} x_{j} \text { s.c. }\left\{\begin{array}{l}
\sum_{i \in V} a_{i} x_{i} \leq b \\
x_{i} \in\{0,1\} \quad i \in V .
\end{array}\right.
$$

Si $E=\varnothing$ c'est-à-dire si la fonction objectif est purement linéaire, on retrouve le problème du sac-à-dos linéaire.

Différentes approches sont possibles pour aborder ce problème : relaxation Lagrangienne [4], décomposition Lagrangienne [3,10], programmation semi-définie [7], linéarisation et méthode polyédrique $[2,8,13,14]$. Dans [5] une linéarisation et une méthode de décomposition sont comparées. On pourra trouver dans [16] un tour d'horizon approfondi de ces diverses méthodes. Ici nous nous intéressons à l'approche polyédrique.

Pour toute paire $(i, j)$ de $E$, posons $y_{i j}=x_{i} x_{j}$ et considérons l'ensemble des solutions admissibles étendues $Q K P(G)=\left\{(x, y) \in\{0,1\}^{|V|+|E|}: \sum_{i \in V} a_{i} x_{i} \leq b\right.$, $\left.y_{i j}=x_{i} x_{j}(i, j) \in E\right\}$ puis l'enveloppe convexe de ces solutions admissibles $P_{Q K P}(G)=\operatorname{Conv}(Q K P(G))$.

$P_{Q K P}(G)$ est le polytope du sac-à-dos quadratique. Si $E=\varnothing$, on retrouve le polytope du sac-à-dos linéaire.

Le problème du sac-à-dos quadratique s'écrit alors comme le problème linéaire suivant :

$$
\max \sum_{i \in V} c_{i} x_{i}+\sum_{(i, j) \in E} c_{i j} y_{i j} \text { s.c. }(x, y) \in P_{Q K P}(G)
$$

car l'optimum de ce problème est atteint en un point de $Q K P(\mathrm{G})$.

Lorsque $b=\sum_{i \in V} a_{i}$, on retrouve le Boolean quadric polytope introduit par Padberg [12] dans le cas du problème quadratique en 0-1 sans contrainte. $P_{Q K P}(G)$ est donc inclus dans le Boolean quadric polytope quelle que soit la valeur de $b$.

Johnson et al. [8] ont introduit le polytope du sac-à-dos quadratique en 1993 pour résoudre le sous-problème d'un algorithme de génération de colonnes pour un problème de partition de graphe. Ils ont proposé comme inégalité valide, l'inégalité 
"arbre" construite sur un sous-arbre de $G$ dont les sommets forment une couverture (c'est-à-dire tel que la somme des poids associés aux sommets dépasse $b$ ). Ils ont montré que cette inégalité induit une facette du polytope du sac-à-dos quadratique défini sur le sous-graphe de $G$ induit par les sommets de l'arbre. Rader [14] a étudié les liftings de cette inégalité pour en faire une facette du polytope du sac-à-dos quadratique défini sur le graphe $G$ tout entier.

Notons aussi le travail de Mehrotra [9] qui a étudié le polytope dans le cas particulier où tous les poids sont égaux à 1 .

Ici nous nous intéressons à la construction de facettes du polytope du sac-à-dos quadratique selon les 2 approches suivantes.

De façon générale, une première approche consiste à se demander sous quelles conditions les facettes du Boolean quadric polytope sont aussi des facettes de $P_{Q K P}(G)$. Des résultats sont donnés pour l'inégalité « coupe »introduite par Padberg [12]. Nous donnons les conditions sous lesquelles cette inégalité induit une facette de $P_{Q K P}(G)$ et quand ces conditions ne sont pas remplies, nous donnons un lifting particulier de l'inégalité qui permet d'en faire une facette de $P_{Q K P}(G)$.

Adams et Sherali [1] ont proposé une linéarisation pour les programmes quadratiques en $0-1$ sous contraintes linéaires obtenue en multipliant les contraintes par les variables $x_{i}$ et leurs compléments $\bar{x}_{i}=1-x_{i}$. Cette approche a beaucoup été utilisée car elle fournit d'assez bonnes relaxations ( $c f$. par exemple $[5,15]$ ). Nous montrons qu'en appliquant cette méthode à des facettes du polytope du sac-à-dos linéaire construit sur le voisinage du sommet $i$, on obtient des facettes de $P_{Q K P}(G)$. Cette approche est ensuite étendue à la multiplication de facettes du sac-à-dos quadratique défini sur le sous-graphe de $G$ induit par le retrait du sommet $i$. Nous donnons des conditions sous lesquelles cette méthode permet de construire des facettes de $P_{Q K P}(G)$. Á titre d'exemple, nous appliquons ces résultats aux inégalités de couverture induisant des facettes du polytope du sacà-dos linéaire et nous obtenons quatre familles de facettes de $P_{Q K P}(G)$.

Finalement nous donnons des résultats numériques illustrant l'utilisation des inégalités ainsi construites dans un algorithme de coupes pour des tailles de problèmes (i.e. cardinal de $V$ ) allant de 50 à 150 variables.

Dans la suite, nous travaillerons toujours sous l'hypothèse

$$
\begin{cases}a_{i} \leq b & \forall i \in V \\ a_{i}+a_{j} \leq b & \forall(i, j) \in E\end{cases}
$$

Noter que la première condition n'est pas impliquée par la seconde dans le cas où $i$ est un sommet isolé de $G$. Sous ces conditions $P_{Q K P}(G)$ est de dimension pleine c'est-à-dire égale à $|V|+|E|[8]$.

Donnons quelques notations et définitions que nous utiliserons pour les graphes.

Pour tout $V^{\prime} \subset V$, on définit $E\left(V^{\prime}\right)=\left\{(i, j) \in E: i, j \in V^{\prime}\right\}$.

Pour tout $V^{\prime}, V^{\prime \prime} \subset V$ disjoints, on définit $\left(V^{\prime}: V^{\prime \prime}\right)=\left\{(i, j) \in E: i \in V^{\prime}\right.$, $\left.j \in V^{\prime \prime}\right\}$.

Pour tout $E^{\prime} \subset E$, on définit $V\left(E^{\prime}\right)=\left\{i \in V: \exists e=(i, j) \in E^{\prime}\right\}$.

Le graphe $\left(V^{\prime}, E\left(V^{\prime}\right)\right)$ est le sous-graphe de $G$ induit par $V^{\prime}$. 
Un graphe partiel de $G=(V, E)$ est un graphe de la forme $\left(V, E^{\prime}\right)$ avec $E^{\prime} \subset E$. Étant donné $a \in V, \Gamma(a)=\{i \in V: \exists e=(a, i) \in E\}$ est l'ensemble des voisins de $a$ et $\delta(a)=\{(a, i) \in E\}$ est l'ensemble des arêtes incidentes à $a$.

Rappelons quelques définitions usuelles concernant les polytopes. Soit $f$ une fonction affine, on dit que l'inégalité $f(x, y) \leq 0$ est valide pour $P_{Q K P}(G)$ si $P_{Q K P}(G) \subset$ $\{(x, y): f(x, y) \leq 0\}$. Noter que l'inclusion $Q K P(\mathrm{G}) \subset\{(x, y): f(x, y) \leq 0\}$ entraîne l'inclusion du polytope par définition de l'enveloppe convexe.

Une inégalité valide $f(x, y) \leq 0$ induit une face de $P_{Q K P}(G)$ à savoir la face définie par $P_{Q K P}(G) \cap(\{(x, y): f(x, y)=0\}$. Une facette est une face propre maximale c'est-à-dire contenue dans aucune autre face hors-mis le polytope luimême.

On notera $u(x, y)=u_{0}+\sum_{i \in V} u_{i} x_{i}+\sum_{(i, j) \in E} u_{i j} y_{i j}=0$ l'équation d'un hyperplan contenant la face induite par une inégalité $f(x, y) \leq 0$ c'est-à-dire t.q. $f(x, y)=0 \Rightarrow u(x, y)=0$ pour tout $(x, y) \in P_{Q K P}(G)$. Le polytope étant de dimension pleine, l'inégalité $f(x, y) \leq 0$ induit une facette de $P_{Q K P}(G)$ ssi il existe un scalaire $\mu$ t.q. $u=\mu f$ ([11], p. 91, Th. 3.6.).

Étant donné $U$ un sous-ensemble de $V$, on notera $(x, y)^{U}$ le point de $Q K P(G)$ t.q. $x_{i}=1$ ssi $i \in U$ et $y_{i j}=1$ ssi $(i, j) \in E(U)$.

\section{InÉGAlité COUPE}

Le polytope du sac-à-dos quadratique étant inclus dans le Boolean quadric polytope pour lequel une bonne connaissance faciale est déjà acquise, il est légitime de se demander sous quelles conditions une facette de ce dernier est aussi une facette du premier et lorsque ces conditions ne sont pas remplies quels sont les liftings permettant d'en faire une facette. Nous donnons des réponses à ces questions dans le cas de l'inégalité « coupe ».

Soient $S, T 2$ sous-ensembles de $V$ non vides et disjoints t.q. le sous-graphe induit par $S \cup T$ soit une clique de $G$. L'inégalité « coupe » est définie par $\operatorname{cut}^{S, T}(x, y)$ $=\sum_{i \in S} x_{i}+\sum_{(i, j) \in E(S)} y_{i j}+\sum_{(i, j) \in E(T)} y_{i j}-\sum_{(i, j) \in(S: T)} y_{i j} \geq 0[12]$.

Les points $(x, y)$ de $Q K P(\mathrm{G})$ qui saturent cette inégalité vérifient l'une des deux conditions, $\sum_{i \in S} x_{i}=\sum_{i \in T} x_{i}$ ou $\sum_{i \in S} x_{i}=\sum_{i \in T} x_{i}-1$.

On suppose $|T| \geq 2$. L'inégalité « coupe » induit une facette du Boolean quadric polytope. Pour le polytope du sac-à-dos quadratique ce n'est plus toujours le cas.

On note $t_{\min 1}, t_{\min 2}, t_{\max 1}, t_{\max 2}$ les indices de $T$ correspondant respectivement aux 2 plus petits des poids de $T$ avec $a_{t_{\min 1}} \leq a_{t_{\min 2}}$ et aux 2 plus grands avec $a_{t_{\max 1}} \geq a_{t_{\max 2}}$.

On note $s_{\min 1}, s_{\max 1}, s_{\max 2}$ les indices de $S$ correspondant respectivement au plus petit des poids de $S$ et pour $|S| \geq 2$ aux 2 plus grands avec $a_{s_{\max 1}} \geq a_{s_{\max 2}}$.

Proposition 2.1. cut $^{S, T}(x, y) \geq 0$ induit une facette de $P_{Q K P}(S \cup T, E(S \cup T))$ ssi

- $C 1: a_{t_{\max 1}}+a_{t_{\max 2}}+a_{s_{\min 1}} \leq b$,

- C2 : $a_{s_{\max 1}}+a_{s_{\max 2}}+a_{t_{\min 1}}+a_{t_{\min 2}} \leq b$ si $|S| \geq 2$. 
Proposition 2.2. cut $^{S, T}(x, y) \geq 0$ induit une facette de $P_{Q K P}(G)$ ssi $C 1, C 2$ de la proposition 2.1 et $C 3: \forall(s, i) \in E$ avec $s \in S$ et $i \in V-S \cup T$

$$
a_{s}+a_{i}+a_{t_{\min }} \leq b .
$$

Démonstration de la proposition 2.1. Notons $F\left(c u t^{S, T}\right)$ la face de $P_{Q K P}(S \cup T$, $E(S \cup T))$ induite par l'inégalité $c^{S} t^{S, T}(x, y) \geq 0$.

Sens suffisant.

Soit $u(x, y)=0$ l'équation d'un hyperplan contenant $F\left(c u t^{S, T}\right)$. Montrons que $u=\mu c u t^{S, T}$.

Les ensembles $U$ suivants sont tels que les points $(x, y)^{U}$ associés appartiennent à $F\left(c u t^{S, T}\right)$ et satisfont donc $u(x, y)^{U}=0$.

Soit $U=\varnothing$, on obtient $u_{0}=0$.

$j \in T$ soit $U=\{j\}$. On obtient $u_{j}=0$.

a) $i \in S, j \in T$ soit $U=\{i, j\}$. On obtient $u_{i}+u_{i j}=0$ soit $u_{i j}=-u_{i}$.

b) $j \in T, k \in T(j \neq k)$ soit $U=\left\{j, k, s_{\min 1}\right\}$. C1 implique l'existence de $(x, y)^{U}$ et on obtient $u_{j k}=u_{s_{\min 1}}$.

Si $|S|=1$ on a finalement $u_{j k}=u_{s_{\min 1}}=-u_{s_{\min 1} j}=\mu \forall j, k \in T(j \neq k)$ et c'est terminé.

Si $|S| \geq 2 . i \in S$ soit $U=\left\{i, t_{\min 1}, t_{\min 2}\right\}$. C2 implique l'existence de $(x, y)^{U}$ et on obtient $u_{t_{\min 1} t_{\min 2}}=u_{i}$.

En prenant $j=t_{\min 1}, k=t_{\min 2}$ dans b) on obtient $u_{i}=u_{s_{\min 1}}$ et finalement $u_{j k}=u_{i}=\mu \quad \forall i \in S, \forall j, k \in T(j \neq k)$. Par a) on obtient $u_{i j}=-\mu \forall i \in S, j \in T$. $i, h \in S$ soit $U=\left\{i, h, t_{\min 1}, t_{\min 2}\right\}$. C2 implique l'existence de $(x, y)^{U}$ et on obtient $u_{i h}=\mu$.

\section{Sens nécessaire.}

Si C1 n'est pas satisfaite alors $F\left(c u t^{S, T}\right)$ est incluse dans l'hyperplan d'équation $y_{t_{\max 1} t_{\max 2}}=0$ et comme $c u t^{S, T} \neq \mu y_{t_{\max 1} t_{\max 2}}, F\left(c u t^{S, T}\right)$ n'est pas une facette de $P_{Q K P}(S \cup T, E(S \cup T))$.

Pour $|S| \geq 2$, si C2 n'est pas satisfaite alors $F\left(c u t^{S, T}\right)$ est incluse dans l'hyperplan d'équation $y_{s_{\max 1} s_{\max 2}}=0$ et comme $c u t^{S, T} \neq \mu y_{s_{\max 1} s_{\max 2}}, F\left(c u t^{S, T}\right)$ n'est pas une facette de $P_{Q K P}(S \cup T, E(S \cup T))$.

Démonstration de la proposition 2.2. Notons $F\left(c^{S} t^{S, T}\right)$ la face de $P_{Q K P}(G)$ induite par l'inégalité $c u t^{S, T}(x, y) \geq 0$.

\section{Sens suffisant.}

Soit $u(x, y)=0$ l'équation d'un hyperplan contenant $F\left(c u t^{S, T}\right)$. Montrons que $u=\mu c u t^{S, T}$.

De la proposition 2.1 on peut déjà déduire que $u(x, y)=\mu c u t^{S, T}(x, y)+$ $\sum_{i \in V-S \cup T} u_{i} x_{i}+\sum_{(i, j) \in E-E(S \cup T)} u_{i j} y_{i j}$. Cherchons les coefficients de $u$ restants. 
Pour cela construisons les ensembles $U$ tels que les points $(x, y)^{U}$ associés appartiennent à $F\left(c u t^{S, T}\right)$ et satisfont donc $u(x, y)^{U}=0$.

$i \in V-S \cup T$ soit $U=\{i\}$. On obtient $u_{i}=0$.

$(t, i) \in E$ avec $t \in T$ et $i \in V-S \cup T$ soit $U=\{t, i\}$. On obtient $u_{t i}=0$.

$(s, i) \in E$ avec $s \in S$ et $i \in V-S \cup T$ soit $U=\left\{s, i, t_{\min 1}\right\}$. C3 entraine l'existence de $(x, y)^{U}$ et on obtient $u_{s i}=0$.

$(i, j) \in E(V-S \cup T)$ soit $U=\{i, j\}$. On obtient $u_{i j}=0$.

Sens nécessaire.

$\mathrm{C} 1, \mathrm{C} 2$ sont nécessaires d'après la proposition 2.1 .

S'il existe une arête $(s, i)$ qui ne satisfait pas C3 alors $F\left(c u t^{S, T}\right)$ est incluse dans l'hyperplan d'équation $y_{s i}=0$ et comme $c u t^{S, T} \neq \mu y_{s i}, F\left(c u t^{S, T}\right)$ n'est pas une facette de $P_{Q K P}(G)$.

On considère une inégalité $c u t^{S, T}(x, y) \geq 0$ qui induit une facette de $P_{Q K P}(S \cup$ $T, E(S \cup T))$ le polytope du sac-à-dos quadratique défini sur le sous-graphe de $G$ induit par $S \cup T$. On s'intéresse alors au plus "grand" graphe $G^{\prime}$ contenu dans $G$ t.q. cut $^{S, T}(x, y) \geq 0$ induise une facette de $P_{Q K P}\left(G^{\prime}\right)$. D'après la proposition 2.2 , $G^{\prime}$ est le graphe $G$ auquel on a retiré les arêtes $(s, i) \in E$ avec $s \in S$ et $i \in V-S \cup T$ qui ne vérifient pas C3 c'est-à-dire t.q. $a_{s}+a_{i}+a_{t_{\min 1}}>b$. On voudrait alors lifter l'inégalité $c u t^{S, T}(x, y) \geq 0$ induisant une facette de $P_{Q K P}\left(G^{\prime}\right)$ pour obtenir une facette de $P_{Q K P}(G)$.

Proposition 2.3. Étant donnée une inégalité cut $^{S, T}(x, y) \geq 0$ qui induit une facette de $P_{Q K P}(S \cup T, E(S \cup T))$, soit $G^{\prime}=\left(V, E^{\prime}\right)$ le graphe partiel de $G$ t.q. $E^{\prime} \subset E$ soit maximal et t.q. cut ${ }^{S, T}(x, y) \geq 0$ induise une facette de $P_{Q K P}\left(G^{\prime}\right)$. Alors il n'existe qu'une inégalité liftée de cut ${ }^{S, T}(x, y) \geq 0$ qui induise une facette de $P_{Q K P}(G)$ à savoir cut ${ }^{S, T}(x, y)-\sum_{e \in E-E^{\prime}} y_{e} \geq 0$.

Pour démontrer cette proposition, nous avons besoin du lemme suivant.

Lemme. Si cut ${ }^{S, T}(x, y) \geq 0$ induit une facette de $P_{Q K P}(S \cup T, E(S \cup T))$ et si $(i, s),(j, s)$ sont 2 arêtes de $G$ avec $i, j$ dans $V-S \cup T$ et $s$ dans $S$ t.q. $a_{s}+a_{i}+a_{t_{\min 1}}>b$ et $a_{s}+a_{j}+a_{t_{\min 1}}>b$ alors $a_{s}+a_{i}+a_{j}>b$.

\section{Démonstration.}

a) Supposons $|S|=1$. $S$ contient un unique élément $s$ qui est aussi $s_{\min 1}$. cut $^{S, T}(x, y) \geq 0$ induisant une facette de $P_{Q K P}(S \cup T, E(S \cup T))$, on a nécessairement $\mathrm{C} 1 a_{t_{\max 1}}+a_{t_{\max 2}}+a_{s} \leq b$

Supposons $a_{s}+a_{i}+a_{j} \leq b(*)$. Alors $a_{s}+a_{i}+a_{t_{\min 1}}>b=>a_{j}<a_{t_{\min 1}} \leq$ $a_{t_{\max 2}}$. En remplaçant dans C1, on obtient $a_{s}+a_{j}+a_{t_{\max 1}} \leq b$ qui est en contracdiction avec $a_{s}+a_{j}+a_{t_{\min 1}}>b$. Donc $(*)$ est impossible.

b) Supposons $|S| \geq 2$. cut $^{S, T}(x, y) \geq 0$ induisant une facette de $P_{Q K P}(S \cup T, E(S \cup T))$, on a nécessairement $\mathrm{C} 2 a_{s_{\max 1}}+a_{s_{\max 2}}+a_{t_{\min 1}}+a_{t_{\min 2}} \leq b$. 


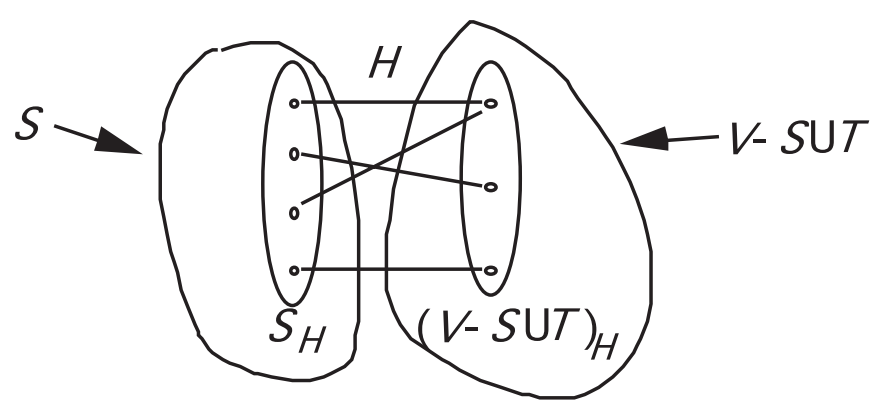

Figure 1. Démonstration de la proposition 2.3 : ensemble $H$ d'arêtes de $E-E^{\prime}$.

Supposons $a_{s}+a_{i}+a_{j} \leq b(*)$. Alors $a_{s}+a_{i}+a_{t_{\min 1}}>b=>a_{j}<a_{t_{\min 1}} \leq a_{t_{\min 2}}$. En remplaçant dans $\mathrm{C} 2$, on obtient $a_{s_{\max 1}}+a_{s_{\max 2}}+a_{t_{\min 1}}+a_{j} \leq b$ qui est en contradiction avec $a_{s}+a_{j}+a_{t_{\min 1}}>b$. Donc $(*)$ est impossible.

Démonstration de la proposition 2.3. On construit les inégalités du polyèdre PL dont les points extrêmes $b \in R^{\left|E-E^{\prime}\right|}$ donnent les liftings de $c u t^{S, T}$ par l'expression $c u t^{S, T}(x, y)+\sum_{e \in E-E^{\prime}} b_{e} y_{e}(c f$. Zemel [17]).

$E^{\prime}$ étant maximal, pour toute arête $(s, i) \in E-E^{\prime}$ on a $a_{s}+a_{i}+a_{t_{\min 1}}>b$ sinon selon C3 (Prop. 2.2) $(s, i)$ serait dans $E^{\prime}$. Soit $H \subset E-E^{\prime}$ t.q. $\sum_{i \in V(H)} a_{i} \leq b$. Notons $S_{H}=V(H) \cap S$ et $(V-S \cup T)_{H}=V(H) \cap(V-S \cup T)$ (cf. Fig. 1).

On a $\sum_{i \in V(H)} a_{i}=\sum_{i \in S_{H}} a_{i}+\sum_{i \in(V-S \cup T)_{H}} a_{i}(*)$. Chaque sommet de $S_{H}$ a un seul voisin dans le graphe $(V(H), H)$ car sinon la somme (*) contiendrait $a_{s}+a_{i}+a_{j}$ avec $(s, i),(s, j) \in H$ et d'après le lemme alors la somme $(*)$ dépasserait strictement $b$.

Notons $i_{s} \in(V-S \cup T)_{H}$ le voisin de $s \in S_{H}$ dans le graphe $(V(H), H)$.

L'inégalité du polyèdre PL associée à $H$ s'écrit :

$$
\begin{aligned}
& \sum_{h \in H} b_{h}+z_{H}^{*} \geq 0 \text { où } z_{H}^{*}=\min \left\{c u t^{S, T}(x, y):\right. \\
& \left.\quad(x, y) \in Q K P\left(G^{\prime}\right) \text { t.q. } x_{i}=1 i \in V(H)\right\} .
\end{aligned}
$$

Étant donné que pour toute arête $(s, i) \in H$ on a $a_{s}+a_{i}+a_{t_{\min 1}}>b$, tout point réalisant le min dans $z_{H}^{*}$ a ses coordonnées $x_{t}$ nulles pour $t \in T$ et finalement $z_{H}^{*}=\frac{1}{2}\left|S_{H}\right|\left(\left|S_{H}\right|+1\right)$.

L'inégalité associée à $H$ est donc $\sum_{s \in S_{H}} b_{s i_{s}}+\frac{1}{2}\left|S_{H}\right|\left(\left|S_{H}\right|+1\right) \geq 0$.

Pour $H=\left\{\left(s, i_{s}\right)\right\}$ de cardinal 1 l'inégalité est $b_{s i_{s}}+1 \geq 0$. Une inégalité associée à un $H$ de cardinal $\geq 2$ est dominée par la somme des inégalités associées aux singletons $\left\{\left(s, i_{s}\right)\right\}$ formés des éléments $\left(s, i_{s}\right)$ de $H$ puisque $\left|S_{H}\right| \leq$ $\frac{1}{2}\left|S_{H}\right|\left(\left|S_{H}\right|+1\right)$. Donc les inégalités associées à un $H$ de cardinal plus grand que 1 peuvent être retirées de la description du polyèdre PL qui est finalement décrit par les inégalités de la forme $b_{s i}+1 \geq 0 \quad(s, i) \in E-E^{\prime}$ et dont l'unique point extrême est le point dont toutes les coordonnées $b_{s i}$ valent -1 . 
Exemple. Soit le problème de sac-à-dos quadratique défini par le graphe $G$ suivant

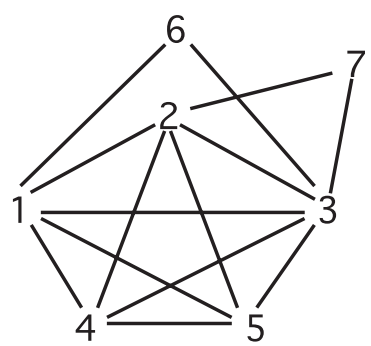

et la contrainte $x_{1}+x_{2}+x_{3}+2 x_{4}+2 x_{5}+6 x_{6}+6 x_{7} \leq 8$.

L'inégalité cut $^{S, T}(x, y) \geq 0$ construite avec $S=\{1,2,3\}, T=\{4,5\}$ induit une facette de $P_{Q K P}\left(G^{\prime}\right)$ où $G^{\prime}$ est le graphe partiel maximal obtenu en enlevant à $G$ les arêtes $(1,6),(3,6),(2,7)$ et $(3,7)$ qui ne satisfont pas C3. Les inégalités du polyèdre $\mathrm{PL}$ ( $c f$. la démonstration de la proposition. 2.3 et [17]) sont :

$$
\begin{array}{ll}
b_{16}+1 \geq 0 & b_{27}+1 \geq 0 \\
b_{36}+1 \geq 0 & b_{37}+1 \geq 0 \\
b_{16}+b_{36}+3 \geq 0 & b_{27}+b_{37}+3 \geq 0
\end{array}
$$

Après élimination des contraintes redondantes, on obtient :

$$
\begin{array}{ll}
b_{16}+1 \geq 0 & b_{27}+1 \geq 0 \\
b_{36}+1 \geq 0 & b_{37}+1 \geq 0
\end{array}
$$

Ce polyèdre comporte un unique point extrême de coordonnées

$$
\begin{array}{ll}
b_{16}=-1 & b_{27}=-1 \\
b_{36}=-1 & b_{37}=-1
\end{array}
$$

et l'unique lifting de l'inégalité « coupe » est cut ${ }^{S, T}(x, y)-y_{16}-y_{36}-y_{27}-y_{37} \geq 0$.

Il est possible d'obtenir d'autres liftings de l'inégalité « coupe » en partant d'un sous-graphe de $G$ plutôt que d'un graphe partiel. Par exemple ici si l'on part du sous-graphe $G^{\prime}$ induit par $S$ et $T$, les inégalités du polyèdre PL sont :

$$
\begin{array}{ll}
b_{6} \geq 0 & b_{7} \geq 0 \\
b_{6}+b_{16}+1 \geq 0 & b_{7}+b_{27}+1 \geq 0 \\
b_{6}+b_{36}+1 \geq 0 & b_{7}+b_{37}+1 \geq 0 \\
b_{6}+b_{16}+b_{36}+3 \geq 0 & b_{7}+b_{27}+b_{37}+3 \geq 0
\end{array}
$$


Ce polyèdre a des points extrêmes t.q. $b_{6} \neq 0$ ou $b_{7} \neq 0$ comme par exemple le point de coordonnées

$$
\begin{array}{ll}
b_{6}=1 & b_{7}=1 \\
b_{16}=-2 & b_{27}=-2 \\
b_{36}=-2 & b_{37}=-2
\end{array}
$$

qui donne le lifting $\mathrm{cut}^{S, T}(x, y)+x_{6}-2 y_{16}-2 y_{36}+x_{7}-2 y_{27}-2 y_{37} \geq 0$.

Comme on le voit sur cet exemple le polyèdre a dans ce cas une structure plus complexe.

\section{Multiplication DE FACETtes DU SAC-À-DOS LinÉaire PAR UNE VARIABLE}

Adams et Sherali [1] proposent une méthode de linéarisation de programme quadratique en 0-1 dans laquelle les contraintes linéaires sont multipliées par les variables et leurs compléments à 1 . Nous allons voir ici que cette approche permet de construire des facettes du polytope du sac-à-dos quadratique à partir de facettes du polytope du sac-à-dos linéaire. Les inégalités construites peuvent être vues comme le produit d'une variable $x_{a}$ ou de son complémenté $\bar{x}_{a}=1-x_{a}$ par une inégalité valide du polytope du sac-à-dos linéaire construit sur le voisinage du sommet $a$.

Étant donné $a \in V$, on définit :

$$
\begin{gathered}
K P^{x_{a}}=\left\{x \in\{0,1\}^{|\Gamma(a)|}: \sum_{i \in \Gamma(a)} a_{i} x_{i} \leq b-a_{a}\right\} \\
K P^{\bar{x}_{a}}=\left\{x \in\{0,1\}^{|\Gamma(a)|}: \sum_{i \in \Gamma(a)} a_{i} x_{i} \leq b\right\}
\end{gathered}
$$

et les polytopes associés $P_{K P}^{x_{a}}=\operatorname{Conv} K P^{x_{a}}$ et $P_{K P}^{\bar{x}_{a}}=\operatorname{Conv} K P^{\bar{x}_{a}}$.

$P_{K P}^{x_{a}}$ et $P_{K P}^{\bar{x}_{a}}$ sont les polytopes construits sur le voisinage du sommet $a$ en fixant resp. $x_{a}=1$ et $x_{a}=0$.

Notons que $P_{K P}^{x_{a}}$ est de dimension pleine. Il en est de même pour $P_{K P}^{x_{a}}$ car $a_{i} \leq b-a_{a} \forall i \in \Gamma(a)$.

\subsection{Construction D'inéGalités valides}

Soit $g(x)=q_{a}+\sum_{i \in \Gamma(a)} q_{i} x_{i} \leq 0$ une inégalité valide pour $P_{K P}^{x_{a}}$ alors l'inégalité $f(x, y)=q_{a} x_{a}+\sum_{i \in \Gamma(a)} q_{i} y_{i a} \leq 0$ est valide pour $P_{Q K P}(G)$. Il suffit de le vérifier sur $Q K P(\mathrm{G})$ :

- si $x_{a}=1$ on a $y_{i a}=x_{i}$ et on retouve $g(x) \leq 0$ qui est valide pour $P_{K P}^{x_{a}}$;

- si $x_{a}=0$ on a $y_{i a}=0$ et on obtient $0 \leq 0$.

Soit $g(x)=q_{a}+\sum_{i \in \Gamma(a)} q_{i} x_{i} \leq 0$ une inégalité valide pour $P_{K P}^{\bar{x}_{a}}$ alors l'inégalité $f(x, y)=q_{a}\left(1-x_{a}\right)+\sum_{i \in \Gamma(a)} q_{i}\left(x_{i}-y_{i a}\right) \leq 0$ est valide pour $P_{Q K P}(G)$. Il suffit de le vérifier sur $Q K P(\mathrm{G})$ :

- si $x_{a}=0$ on a $y_{i a}=0$ et on retouve $g(x) \leq 0$ qui est valide pour $P_{K P}^{\bar{x}_{a}}$; 
- si $x_{a}=1$ on a $y_{i a}=x_{i}$ et on obtient $0 \leq 0$.

L'inégalité $f(x, y) \leq 0$ peut s'interpréter comme étant la linéarisation de l'inégalité obtenue resp. par les produits $x_{a} \times g(x) \leq 0$ et $\left(1-x_{a}\right) \times g(x) \leq 0$.

Ces inégalités induisent des facettes à condition de partir d'inégalités induisant des facettes.

Proposition 3.1. $f(x, y)=q_{a} x_{a}+\sum_{i \in \Gamma(a)} q_{i} y_{i a} \leq 0$ induit une facette de $P_{Q K P}(G)$ ssi $g(x)=q_{a}+\sum_{i \in \Gamma(a)} q_{i} x_{i} \leq 0$ induit une facette de $P_{K P}^{x_{a}}$.

Proposition 3.2. Sous l'hypothèse $H=\left\{\begin{array}{ll}a_{a}+a_{i} \leq b & i \in V-\{a\} \\ a_{a}+a_{i}+a_{j} \leq b & (i, j) \in E-\delta(a)\end{array}\right.$, $f(x, y)=q_{a}\left(1-x_{a}\right)+\sum_{i \in \Gamma(a)} q_{i}\left(x_{i}-y_{i a}\right) \leq 0$ induit une facette de $P_{Q K P}(G)$ ssi $g(x)=q_{a}+\sum_{i \in \Gamma(a)} q_{i} x_{i} \leq 0$ induit une facette de $P_{K P}^{\bar{x}_{a}}$.

Démonstration de la proposition 3.1.

Sens suffisant.

Soit $u(x, y)=0$ l'équation d'un hyperplan contenant la face induite par l'inégalité $f(x, y) \leq 0$.

Les points $(x, y)^{U}$ définis par les ensembles $U$ suivants, sont dans $Q K P(G)$ et satisfont l'égalité $f(x, y)=0$ et donc $u(x, y)=0$.

Avec $U=\emptyset$, on obtient $u_{0}=0$.

Avec $U=\{i\} \quad i \in V-\{a\}$, on obtient $u_{0}+u_{i}=0$ et finalement $u_{i}=0$.

Avec $U=\{i, j\} \quad(i, j) \in E-\delta(a)$, on obtient $u_{0}+u_{i}+u_{j}+u_{i j}=0$ et finalement $u_{i j}=0$.

Notons $\hat{x}$ les points de $K P^{x_{a}}$ satisfaisant l'égalité $g(\hat{x})=0$. Considérons maintenant les points $(x, y)$ de $Q K P(\mathrm{G})$ définis par

$$
\begin{cases}x_{a}=1 & \\ x_{i}=\hat{x}_{i} & i \in \Gamma(a) \\ x_{i}=0 & i \notin\{a\} \cup \Gamma(a) .\end{cases}
$$

Alors $y_{i a}=\hat{x}_{i}$ pour tout $i \in \Gamma(a)$. Ces points satisfont $f(x, y)=0$ et donc $u(x, y)=u_{a}+\sum_{i \in \Gamma(a)} u_{i a} y_{i a}=u_{a}+\sum_{i \in \Gamma(a)} u_{i a} \hat{x}_{i}=0$. Comme l'inégalité $g(x) \leq 0$ induit une facette de $P_{K P}^{x_{a}}$, il existe $\mu$ t.q. $u_{a}=\mu q_{a}$ et $u_{i a}=\mu q_{i}$. Finalement $u=\mu f$.

Démonstration de la proposition 3.2.

Sens suffisant.

Soit $u(x, y)=0$ l'équation d'un hyperplan contenant la face induite par l'inégalité $f(x, y) \leq 0$. Les points $(x, y)^{U}$ définis par les ensembles $U$ suivants, sont sous l'hypothèse $H$, dans $Q K P(G)$ et satisfont l'égalité $f(x, y)=0$ et donc $u(x, y)=0$.

Avec $U=\{a\}$, on obtient $u_{0}+u_{a}=0$.

Avec $U=\{a, i\} \quad i \in V-\{a\}$, on obtient $u_{0}+u_{a}+u_{i}+u_{i a}=0$ et finalement $u_{i}=-u_{i a}$ en posant $u_{i a}=0$ si $(i, a) \notin E$. 
Avec $U=\{a, i, j\}(i, j) \in E-\delta(a)$, on obtient $u_{0}+u_{a}+u_{i}+u_{j}+u_{i a}+u_{j a}+u_{i j}=0$ et finalement $u_{i j}=0$.

Notons $\hat{x}$ les points de $K P^{\bar{x}_{a}}$ satisfaisant l'égalité $g(\hat{x})=0$. Considérons maintenant les points $(x, y)$ de $Q K P(\mathrm{G})$ définis par

$$
\left\{\begin{array}{l}
x_{a}=0 \\
x_{i}=\hat{x}_{i} \quad i \in \Gamma(a) \\
x_{i}=0 \quad i \notin\{a\} \cup \Gamma(a) .
\end{array}\right.
$$

Alors $y_{i a}=0$ pour tout $i \in \Gamma(a)$. Ces points satisfont $f(x, y)=0$ et donc $u(x, y)=$ $u_{0}+\sum_{i \in \Gamma(a)} u_{i} \hat{x}_{i}=-u_{a}-\sum_{i \in \Gamma(a)} u_{i a} \hat{x}_{i}=0$. Comme l'inégalité $g(x) \leq 0$ induit une facette de $P_{K P}^{\bar{x}_{a}}$, il existe $\mu$ t.q. $u_{0}=-u_{a}=\mu q_{a}$ et $u_{i}=-u_{i a}=\mu q_{i}$ pour tout $i \in \Gamma(a)$. Finalement $u=\mu f$.

Démonstration des propositions 3.1 et 3.2 .

Sens nécessaire.

Supposons que l'inégalité $g(x) \leq 0$ n'induise pas une facette de $P_{K P}^{x_{a}}\left(\right.$ resp. $\left.P_{K P}^{\bar{x}_{a}}\right)$. Si $g=0$ alors $f=0$ et la face induite par l'inégalité $f(x, y) \leq 0$ n'est autre que $P_{Q K P}(G)$. Supposons $g \neq 0$ alors il existe $g^{\prime}(x)=q_{a}^{\prime}+\sum_{i \in \Gamma(a)} q_{i}^{\prime} x_{i} \neq \mu g(x)$ pour tout réel $\mu$ t.q. la face de $P_{K P}^{x_{a}}$ (resp. $P_{K P}^{\bar{x}_{a}}$ ) induite par l'inégalité $g(x) \leq 0$ est contenue dans l'hyperplan d'équation $g^{\prime}(x)=0$. Alors la face de $P_{Q K P}(G)$ induite par l'inégalité $f(x, y) \leq 0$ est contenue dans l'hyperplan d'équation $f^{\prime}(x, y)=$ $q_{a}^{\prime} x_{a}+\sum_{i \in \Gamma(a)} q_{i}^{\prime} y_{i a}=0\left(\right.$ resp. $\left.f^{\prime}(x, y)=q_{a}^{\prime}\left(1-x_{a}\right)+\sum_{i \in \Gamma(a)} q_{i}^{\prime}\left(x_{i}-y_{i a}\right)=0\right)$ et comme $f^{\prime} \neq \mu f$ pour tout réel $\mu$ l'inégalité $f(x, y) \leq 0$ n'induit pas une facette de $P_{Q K P}(G)$

\subsection{Applichtion À L'InÉgalité De COUverture}

Les inégalités de couvertures sont souvent utilisées pour la résolution du sac-àdos linéaire ( $c f$. par exemple la publication récente [6]). Nous donnons ici quelques rappels sur cette inégalité puis nous appliquons les précédentes propositions.

Considérons un ensemble $N$ de $n$ indices et le polytope du sac-à-dos linéaire défini par $P_{N, b}=\operatorname{Conv}\left\{x \in\{0,1\}^{n}: \sum_{i \in N} a_{i} x_{i} \leq b\right\}$ avec $0 \leq a_{i} \leq b i \in N$.

Soit un entier $\alpha$ positif et $S \subset N$ t.q. $|S| \geq \alpha+1$. Maintenant supposons que tout $S^{\prime} \subset S$ t.q. $\left|S^{\prime}\right| \geq \alpha+1$ soit une couverture c'est-à-dire vérifie $\sum_{i \in S^{\prime}} a_{i}>b$ alors l'inégalité $\sum_{i \in S} x_{i} \leq \alpha$ est valide pour $P_{N, b}$. Cette inégalité induit une facette de $P_{N, b}$ sous certaines conditions.

Pour simplifier les notations, supposons $S=\{1,2, \cdots, s\}$ et $a_{1} \leq a_{2} \leq \cdots \leq a_{s}$. L'inégalité $\sum_{i \in S} x_{i} \leq \alpha$ induit une facette de $P_{N, b}$ ssi

$$
\left\{\begin{array}{ll}
a_{1}+\cdots+a_{\alpha-1}+a_{s} \leq b & \left(\mathrm{C}_{1}\right) \\
a_{2}+\cdots+a_{\alpha+1} \leq b & \left(\mathrm{C}_{2}\right) \\
a_{1}+\cdots+a_{\alpha}+\max _{i \in N-S} a_{i} \leq b & \left(\mathrm{C}_{3}\right)
\end{array} \quad \text { (par convention } a_{1}+\cdots+a_{0}=0\right) .
$$


Les ensembles $S$ satisfaisant ces conditions sont des extensions de couverture minimale avec $C=\{1, \ldots, \alpha+1\}$ la couverture minimale et $S=E(C)=$ $C \cup\left\{i \in N \backslash C\right.$ t.q. $\left.a_{i} \geq a_{j} \forall j \in C\right\}$ son extension (cf. [11] p. 266).

Pour $S$ et $\alpha$ vérifiant les définitions ci-dessus, on dira que $S$ est une $(\alpha+1)$ configuration de $P_{N, b}$.

À la lumière de ces rappels, appliquons les propositions 3.1 et 3.2 pour obtenir des familles de facettes de $P_{Q K P}(G)$.

Soit $t \in V, S \subset \Gamma(t)$ et un entier positif $\alpha$ t.q. $S$ soit une $(\alpha+1)$-configuration du polytope $P_{K P}^{x_{t}}$, alors l'inégalité $\sum_{i \in S} y_{i t} \leq \alpha x_{t}$ est valide pour $P_{Q K P}(G)$ (cf. Sect. 3.1). De plus nous avons le résultat suivant.

Proposition 3.2.1. Supposons $S=\{1,2, \cdots, s\}$ et $a_{1} \leq a_{2} \leq \cdots \leq a_{s}$ ( $s \geq$ $\alpha+1)$, alors l'inégalité $\sum_{i \in S} y_{i t} \leq \alpha x_{t}$ induit une facette de $P_{Q K P}(G)$ ssi

$$
\begin{cases}a_{1}+\cdots+a_{\alpha-1}+a_{s} \leq b-a_{t} & \left(C_{1.1}\right) \\ a_{2}+\cdots+a_{\alpha+1} \leq b-a_{t} & \left(C_{1.2}\right) \\ a_{1}+\cdots+a_{\alpha}+\max _{i \in \Gamma(t)-S} a_{i} \leq b-a_{t} . & \left(C_{1.3}\right)\end{cases}
$$

Démonstration. D'après le rappel, $\sum_{i \in S} x_{i} \leq \alpha$ induit une facette pour le polytope du sac-à-dos linéaire $P_{K P}^{x_{t}}=P_{\Gamma(t), b-a_{t}} \mathrm{ssi}_{1.1}, \mathrm{C}_{1.2}, \mathrm{C}_{1.3}$. On applique alors la proposition 3.1 .

On retrouve l'inégalité introduite par Rader [13] et qui avait montré qu'elle induit une facette du polytope du sac-à-dos quadratique construit sur le sousgraphe de $G$ induit par $S \cup\{t\}$. Pour $|S|=\alpha+1$, on retrouve l'inégalité "étoile" introduite par Johnson et al. [8].

On dira que le couple $t, S$ est une $(1, \alpha+1)$-configuration de $P_{Q K P}(G)$.

Maintenant supposons que $S$ soit une $(\alpha+1)$-configuration du polytope $P_{K P}^{\bar{x}_{t}}$, alors l'inégalité $\sum_{i \in S}\left(x_{i}-y_{i t}\right) \leq \alpha\left(1-x_{t}\right)$ est valide pour $P_{Q K P}(G)$ (cf. Sect. 3.1). De plus nous avons le résultat suivant.

Proposition 3.2.2. Soit la condition $H=\left\{\begin{array}{ll}a_{t}+a_{i} \leq b & i \in V-\{t\} \\ a_{t}+a_{i}+a_{j} \leq b & (i, j) \in E-\delta(t)\end{array}\right.$. Supposons $S=\{1,2, \cdots, s\}$ et $a_{1} \leq a_{2} \leq \cdots \leq a_{s}(s \geq \alpha+1)$ et $H$ vérifiée, alors l'inégalité $\sum_{i \in S}\left(x_{i}-y_{i t}\right) \leq \alpha\left(1-x_{t}\right)$ induit une facette de $P_{Q K P}(G)$ ssi

$$
\begin{cases}a_{1}+\cdots+a_{\alpha-1}+a_{s} \leq b & \left(C_{2.1}\right) \\ a_{2}+\cdots+a_{\alpha+1} \leq b & \left(C_{2.2}\right) \\ a_{1}+\cdots+a_{\alpha}+\max _{i \in \Gamma(t)-S} a_{i} \leq b . & \left(C_{2.3}\right)\end{cases}
$$

Démonstration. D'après le rappel $\sum_{i \in S} x_{i} \leq \alpha$ induit une facette pour le polytope du sac-à-dos linéaire $P_{K P}^{\bar{x}_{t}}=P_{\Gamma(t), b}$ ssi $\mathrm{C}_{2.1}, \mathrm{C}_{2.2}, \mathrm{C}_{2.3}$. On applique alors la proposition 3.2.

On dira que le couple $t, S$ est une $(0, \alpha+1)$-configuration de $P_{Q K P}(G)$. 


\section{Multiplication de facettes DU SaC-À-Dos Quadratique PAR UNE VARIABLE}

Les propositions 4.1 et 4.2 qui suivent, permettent de construire de nouvelles inégalités induisant des facettes de $P_{Q K P}(G)$ à partir d'inégalités induisant des facettes d'un polytope $P_{Q K P}\left(G^{\prime}\right)$ défini sur un sous-graphe $G^{\prime}$ de $G$.

Plus précisément, les inégalités construites peuvent être vues comme le produit d'une variable $x_{a}$ ou de son complémenté $\bar{x}_{a}=1-x_{a}$ par une inégalité valide du polytope induit par le sous-graphe $G-\{a\}$, les termes cubiques $z_{a i j}=x_{a} x_{i} x_{j}$ engendrés étant implicitement éliminés à l'aide des inégalités de la forme $z_{a i j} \leq$ $y_{a i}, z_{a i j} \leq y_{a j}$ ou $z_{a i j} \leq y_{i j}$.

Étant donné $a \in V$, on considère $G-\{a\}=(V-\{a\}, E(V-\{a\}))$ le sousgraphe de $G$ induit par le retrait de $a$ et on définit :

$$
\begin{aligned}
Q K P^{x_{a}}(G)= & \left\{(x, y) \in\{0,1\}^{|V|-1+|E|-|\delta(a)|}:\right. \\
& \left.\sum_{i \in V-\{a\}} a_{i} x_{i} \leq b-a_{a}, y_{i j}=x_{i} x_{j} \quad(i, j) \in E(V-\{a\})\right\} \\
Q K P^{\bar{x}_{a}}(G)=\{ & (x, y) \in\{0,1\}^{|V|-1+|E|-|\delta(a)|}: \\
& \left.\sum_{i \in V-\{a\}} a_{i} x_{i} \leq b, y_{i j}=x_{i} x_{j} \quad(i, j) \in E(V-\{a\})\right\}
\end{aligned}
$$

et les polytopes associés $P_{Q K P}^{x_{a}}(G)=\operatorname{Conv} Q K P^{x_{a}}(G)$ et $P_{Q K P}^{\bar{x}_{a}}(G)=$ $\operatorname{Conv} Q K P^{\bar{x}_{a}}(G)$.

Les points extrêmes $Q K P^{x_{a}}(G)$, resp. $Q K P^{\bar{x}_{a}}(G)$, de ces polytopes sont les points de $\operatorname{QKP}(\mathrm{G})$ tels que $x_{a}=1$, resp. $x_{a}=0$, et que l'on projète sur les variables $x$ indexées dans $V-\{a\}$ et les variables $y$ indexées dans $E-\delta(a)$.

\subsection{Multiplication D'Une facette par une variable Directe}

Soit $a \in V, V^{\prime} \subset \Gamma(a), E^{\prime} \subset E-\delta(a)$, où $V^{\prime}, E^{\prime}$ est le support de l'inégalité valide pour $P_{Q K P}^{x_{a}}(G)$ :

$$
f(x, y)=q_{a}+\sum_{i \in V^{\prime}} q_{i} x_{i}+\sum_{(i, j) \in E^{\prime}} q_{i j} y_{i j} \geq 0
$$

avec $q_{i j}>0 \forall(i, j) \in E^{\prime}, q_{i} \neq 0 \quad \forall i \in V^{\prime}$.

L'inégalité $h(x, y)=q_{a} x_{a}+\sum_{i \in V^{\prime}} q_{i} y_{i a}+\sum_{(i, j) \in E^{\prime}} q_{i j} y_{i j} \geq 0$ est valide pour $P_{Q K P}(G)$. Il suffit de le vérifier sur $Q K P(G)$ :

- si $x_{a}=1$ on retouve $f(x, y) \geq 0$ qui est valide pour $P_{Q K P}^{x_{a}}(G)$;

- si $x_{a}=0$ on obtient $\sum_{(i, j) \in E^{\prime}} q_{i j} y_{i j} \geq 0$ qui est valide car $q_{i j}>0 \quad \forall(i, j) \in E^{\prime}$. 
Proposition 4.1. Sous l'hypothèse $H=\left\{\begin{array}{ll}a_{a}+a_{i} \leq b & i \in V-\{a\} \\ a_{a}+a_{i}+a_{j} \leq b & (i, j) \in E-\delta(a)\end{array}\right.$, si $f(x, y)=q_{a}+\sum_{i \in V^{\prime}} q_{i} x_{i}+\sum_{(i, j) \in E^{\prime}} q_{i j} y_{i j} \geq 0$ induit une facette de $P_{Q K P}^{x_{a}}(G)$ alors $h(x, y)=q_{a} x_{a}+\sum_{i \in V^{\prime}} q_{i} y_{i a}+\sum_{(i, j) \in E^{\prime}} q_{i j} y_{i j} \geq 0$ induit une facette de $P_{Q K P}(G)$.

Démonstration. Soit $u(x, y)=0$ l'équation d'un hyperplan contenant la face induite par l'inégalité $h(x, y) \geq 0$.

On considère d'abord les points $(x, y)$ de $Q K P(\mathrm{G})$ t.q. $x_{a}=1$.

Alors $y_{i a}=x_{i}$ pour tout $i \in \Gamma(a)$ et $u(x, y)=0$ devient $u(x, y)=u_{0}+u_{a}+$ $\sum_{i \in V-\{a\}} u_{i} x_{i}+\sum_{i \in \Gamma(a)} u_{i a} x_{i}+\sum_{(i, j) \in E-\delta(a)} u_{i j} y_{i j}=0$. Cet hyperplan contient la facette de $P_{Q K P}^{x_{a}}(G)$ induite par $f(x, y) \geq 0$. Sous l'hypothèse $H$ le polytope $P_{Q K P}^{x_{a}}(G)$ est de dimension pleine et cela entraine qu'il existe $\mu$ t.q. :

$u_{0}+u_{a}=\mu q_{a}$

$u_{i}+u_{i a}=\mu q_{i}$ pour tout $i \neq a$, en posant $u_{i a}=0$ si $(i, a) \notin E$ et $q_{i}=0$ si $i \notin$ $V^{\prime}$;

$u_{i j}=\mu q_{i j}$ pour tout $(i, j) \in E-\delta(a)$, en posant $q_{i j}=0$ si $(i, j) \notin E^{\prime}$.

On considère maintenant les points $(x, y)^{U}$ de $Q K P(\mathrm{G})$ t.q. $x_{a}=0$ et satisfaisant l'égalité $h(x, y)=0$ et donc $u(x, y)=0$.

Avec $U=\emptyset$, on obtient $u_{0}=0$.

Avec $U=\{i\} i \in V-\{a\}$, on obtient $u_{0}+u_{i}=0$ et finalement $u_{i}=0$.

Ce qui entraine finalement que $u=\mu h$.

Nous allons maintenant appliquer les résultats précédents.

Soit $t, S$ une $(0, \alpha+1)$-configuration du polytope $P_{Q K P}^{x_{a}}(G)$ où $a \in V-(S \cup\{t\})$ et $S \cup\{t\} \subset \Gamma(a)$.

L'inégalité $f(x, y)=\alpha\left(1-x_{t}\right)+\sum_{i \in S}\left(y_{i t}-x_{i}\right) \geq 0$ est valide sur $P_{Q K P}^{x_{a}}(G)$ (cf. Sect. 3.2). Alors $h(x, y)=\alpha\left(x_{a}-y_{a t}\right)+\sum_{i \in S}\left(y_{i t}-y_{i a}\right) \geq 0$ est valide sur $P_{Q K P}(G)$.

De plus on a le résultat suivant.

Proposition 4.1.1. Soit $S=\{1, \cdots, s\}$ avec $a_{1} \leq \cdots \leq a_{s}$ et $s \geq \alpha+1$. Alors, si

$$
\left(C_{2.0}\right)= \begin{cases}a_{t}+a_{i} \leq b-a_{a} & i \in V-\{t\}-\{a\} \\ a_{t}+a_{i}+a_{j} \leq b-a_{a} & (i, j) \in E-\delta(t)-\delta(a)\end{cases}
$$

est satisfaite et si

$$
\left\{\begin{array}{lr}
a_{1}+\cdots+a_{\alpha-1}+a_{s} \leq b-a_{a} & \left(C_{2.1}\right) \\
a_{2}+\cdots+a_{\alpha+1} \leq b-a_{a} & \left(C_{2.2}\right) \\
a_{1}+\cdots+a_{\alpha}+\max _{i \in \Gamma(t)-S-\{a\}} a_{i} \leq b-a_{a} & \left(C_{2.3}\right)
\end{array}\right.
$$

l'inégalité $h(x, y)=\alpha\left(x_{a}-y_{a t}\right)+\sum_{i \in S}\left(y_{i t}-y_{i a}\right) \geq 0$ induit une facette de $P_{Q K P}(G)$. 
Démonstration. Les conditions $\left(\mathrm{C}_{2.0}, \mathrm{C}_{2.1}, \mathrm{C}_{2.2}, \mathrm{C}_{2.3}\right)$ impliquent que l'inégalité $f(x, y)=\alpha\left(1-x_{t}\right)+\sum_{i \in S}\left(y_{i t}-x_{i}\right) \geq 0$ induit une facette de $P_{Q K P}^{x_{a}}(G)(c f$. Sect. 3.2, Prop. 3.2.2).

De plus $\left(\mathrm{C}_{2.0}\right)$ implique l'hypothèse $H$ de la proposition 4.1. que l'on applique alors.

\subsection{Multiplication D'une facette par une variable complémentée}

Soit $a \in V, V^{\prime} \subset \Gamma(a), E^{\prime} \subset E-\delta(a)$ t.q. $\forall(i, j) \in E^{\prime} \quad\{i, j\} \cap \Gamma(a) \neq \varnothing$, où $V^{\prime}, E^{\prime}$ est le support de l'inégalité valide pour $P_{Q K P}^{\bar{x}_{a}}(G)$ :

$$
f(x, y)=q_{a}+\sum_{i \in V^{\prime}} q_{i} x_{i}+\sum_{(i, j) \in E^{\prime}} q_{i j} y_{i j} \leq 0
$$

avec $q_{i j}>0 \forall(i, j) \in E^{\prime}, q_{i} \neq 0 \quad \forall i \in V^{\prime}$.

Pour chaque $(i, j) \in E^{\prime}$, on choisit une extrémité $(i$ ou $j)$, notée $\rho_{i j}$, qui est dans $\Gamma(a)$.

L'inégalité $h^{\rho}(x, y)=q_{a}\left(1-x_{a}\right)+\sum_{i \in V^{\prime}} q_{i}\left(x_{i}-y_{i a}\right)+\sum_{(i, j) \in E^{\prime}} q_{i j}\left(y_{i j}-y_{a \rho_{i j}}\right)$ $\leq 0$ est valide pour $P_{Q K P}(G)$. Il suffit de le vérifier sur $Q K P(G)$ :

- si $x_{a}=0$ on retouve $f(x, y) \leq 0$ qui est valide pour $P_{Q K P}^{\bar{x}_{a}}(G)$;

- si $x_{a}=1$ on obtient $\sum_{(i, j) \in E^{\prime}} q_{i j}\left(y_{i j}-x_{\rho_{i j}}\right) \leq 0$ qui est valide car $q_{i j}>$ $0 \forall(i, j) \in E^{\prime}$ et $y_{i j} \leq x_{\rho_{i j}}$.

Proposition 4.2. Soit $F$ la face de $P_{Q K P}^{x_{a}}(G)$ induite par l'inégalité $\sum_{(i, j) \in E^{\prime}} q_{i j} \times$ $\left(y_{i j}-x_{\rho_{i j}}\right) \leq 0$. Si $f(x, y)=q_{a}+\sum_{i \in V^{\prime}} q_{i} x_{i}+\sum_{(i, j) \in E^{\prime}} q_{i j} y_{i j} \leq 0$ induit une facette de $P_{Q K P}^{\bar{x}_{a}}(G)$ et si la projection de $F$ sur les variables $x_{i \in \Gamma(a)}$ est de dimension pleine (c'est-à-dire égale à $|\Gamma(a)|)$ alors $h^{\rho}(x, y)=q_{a}\left(1-x_{a}\right)+$ $\sum_{i \in V^{\prime}} q_{i}\left(x_{i}-y_{i a}\right)+\sum_{(i, j) \in E^{\prime}} q_{i j}\left(y_{i j}-y_{a \rho_{i j}}\right) \leq 0$ induit une facette de $P_{Q K P}(G)$.

Démonstration. Soit $u(x, y)=0$ l'équation d'un hyperplan contenant la face induite par l'inégalité $h^{\rho}(x, y) \leq 0$.

On considère d'abord les points $(x, y)$ de $Q K P(\mathrm{G})$ t.q. $x_{a}=0$. Alors $y_{i a}=$ 0 pour tout $i \in \Gamma(a)$ et $u(x, y)=0$ devient $u(x, y)=u_{0}+\sum_{i \in V-\{a\}} u_{i} x_{i}+$ $\sum_{(i, j) \in E-\delta(a)} u_{i j} y_{i j}=0$. Cet hyperplan contient la facette de $P_{Q K P}^{\bar{x}_{a}}(G)$ induite par $f(x, y) \leq 0$. Comme $P_{Q K P}^{\bar{x}_{a}}(G)$ est de dimension pleine, cela entraine qu'il existe $\mu$ tel que :

$$
\begin{aligned}
& u_{0}=\mu q_{a} ; \\
& u_{i}=\mu q_{i} \text { pour tout } i \neq a, \text { en posant } q_{i}=0 \text { si } i \notin V^{\prime} ; \\
& u_{i j}=\mu q_{i j} \text { pour tout }(i, j) \in E-\delta(a), \text { en posant } q_{i j}=0 \text { si }(i, j) \notin E^{\prime} .
\end{aligned}
$$

On considère maintenant les points $(x, y)$ de $Q K P(\mathrm{G})$ t.q. $x_{a}=1$ et satisfaisant l'égalité $h^{\rho}(x, y)=0$ et donc $u(x, y)=0$. 
Comme $y_{i a}=x_{i}$ pour tout $i \in \Gamma(a), h^{\rho}(x, y)=0$ devient $\sum_{(i, j) \in E^{\prime}} q_{i j}\left(y_{i j}-x_{\rho_{i j}}\right)$ $=0(2)$ et $u(x, y)=0$ devient

$$
u(x, y)=\mu q_{a}+u_{a}+\sum_{i \in V^{\prime}} \mu q_{i} x_{i}+\sum_{i \in \Gamma(a)} u_{i a} x_{i}+\sum_{(i, j) \in E^{\prime}} \mu q_{i j} y_{i j}=0 .
$$

On peut voir (1) comme un système d'équations avec autant d'égalités qu'il y a de points $(x, y)$ de $Q K P(\mathrm{G})$ t.q. $x_{a}=1$ et $h^{\rho}(x, y)=0$, et dont les inconnues sont $u_{a}$ et $u_{i a} i \in \Gamma(a)$. Les coefficients des inconnues sont respectivement 1 et $x_{i} i \in \Gamma(a)$. Par définition les points de $F$ vérifient (2) et donc (1) et par hypothèse la projection de $F$ sur les variables $x_{i} i \in \Gamma(a)$ est de dimension pleine ; cela revient à dire que le rang des vecteurs $\left(1, x_{i \in \Gamma(a)}\right)$ est $|\Gamma(a)|+1$ et donc le système (1) admet une solution unique. Nécessairement celle-ci est donné par $u=\mu h^{\rho}$ car alors $h^{\rho}(x, y)=0 \Rightarrow u(x, y)=0$ et (1) est vérifié.

Nous allons maintenant appliquer les résultats précédents.

Soit $t, S$ une $(1, \alpha+1)$-configuration du polytope $P_{Q K P}^{\bar{x}_{a}}(G)$ où $a \in V-(S \cup\{t\})$ et $t \in \Gamma(a)$.

L'inégalité $f(x, y)=\sum_{i \in S} y_{i t}-\alpha x_{t} \leq 0$ est valide sur $P_{Q K P}^{\bar{x}_{a}}(G)$ (cf. Sect. 3.2). Soit $S^{\prime} \subset S \cap \Gamma(a)$ et $S^{\prime \prime}=S-S^{\prime}$ alors $h(x, y)=\sum_{i \in S^{\prime}}\left(y_{i t}-y_{a i}\right)+$ $\sum_{i \in S^{\prime \prime}}\left(y_{i t}-y_{a t}\right)-\alpha\left(x_{t}-y_{a t}\right) \leq 0$ est valide sur $P_{Q K P}(G)$. En effet, en reprenant les notations précédentes, les arêtes $E^{\prime}$ sont ici de la forme $(t, i)$ avec $i \in S$ et si on choisit $\rho_{t i}=i$ si $i \in S^{\prime}$ et $\rho_{t i}=t$ si $i \in S^{\prime \prime}$, on obtient $h^{\rho}(x, y) \leq 0$ qui, comme on l'a vu plus haut, est valide sur $P_{Q K P}(G)$.

De plus nous avons le résultat suivant.

Proposition 4.2.1. Soit $S=\{1, \cdots, s\}$ avec $a_{1} \leq \cdots \leq a_{s}$ et $s \geq \alpha+1$. Alors, si

$$
\left\{\begin{array}{lr}
a_{1}+\cdots+a_{\alpha-1}+a_{s} \leq b-a_{t} & \left(C_{1.1}\right) \\
a_{2}+\cdots+a_{\alpha+1} \leq b-a_{t} & \left(C_{1.2}\right) \\
a_{1}+\cdots+a_{\alpha}+\max _{i \in \Gamma(t)-S-\{a\}} a_{i} \leq b-a_{t} & \left(C_{1.3}\right) \\
a_{t}+\max _{i \in S^{\prime}} a_{i}+\sum_{i \in S^{\prime \prime}} a_{i} \leq b-a_{a} & \left(C_{1.4}\right)
\end{array}\right.
$$

l'inégalité $h(x, y)=\sum_{i \in S^{\prime}}\left(y_{i t}-y_{a i}\right)+\sum_{i \in S^{\prime \prime}}\left(y_{i t}-y_{a t}\right)-\alpha\left(x_{t}-y_{a t}\right) \leq 0$ induit une facette de $P_{Q K P}(G)$.

Démonstration. Les conditions $\left(\mathrm{C}_{1.1}, \mathrm{C}_{1.2}, \mathrm{C}_{1.3}\right)$ entrainent que l'inégalité $f(x, y)$ $=\sum_{i \in S} y_{i t}-\alpha x_{t} \leq 0$ induit une facette de $P_{Q K P}^{\bar{x}_{a}}(G)$ (cf. Sect. 3.2, Prop. 3.1.1).

Exhibons $|\Gamma(a)|+1$ points de $Q K P^{x_{a}}(G)$ vérifiant $\sum_{i \in S^{\prime}}\left(y_{i t}-x_{i}\right)+$ $\sum_{i \in S^{\prime \prime}}\left(y_{i t}-x_{t}\right)=0$ et t.q. la projection sur les variables $x_{i \in \Gamma(a)}$ donne une famille affinement indépendante.

La condition $\left(\mathrm{C}_{1.4}\right)$ permet de construire les points $(x, y)^{U}$ de $Q K P^{x_{a}}(G)$ définis par les sous-ensembles $U$ de $V-\{a\}$ suivants : 


$$
\begin{cases}U=\{i, t\} \cup S^{\prime \prime} & i \in S^{\prime} \\ U=\{t\} \cup S^{\prime \prime} & \\ U=\{i\} & i \in \Gamma(a)-\left(S^{\prime} \cup\{t\}\right) \\ U=\varnothing . & \end{cases}
$$

On vérifie aisément que la projection sur les variables $x_{i \in \Gamma(a)}$ des points construits ci-dessus forme une famille affinement indépendante.

On applique alors la proposition 4.2 .

\section{RÉsultats NUMÉRIQUeS}

Les inégalités présentées dans les sections 2 à 4 ont été mises en oeuvre dans un algorithme de coupes. Le but est ici d'évaluer la contribution de ces inégalités à la résolution du problème de sac-à-dos quadratique en 0-1 par la méthode polyédrique.

\subsection{Présentation de L'Algorithme de COUPES}

L'algorithme de coupes a la structure classique suivante : soit $P_{0}$ un polyèdre initial contenant $P_{Q K P}(G)$. Poser $P=P_{0}$.

1. Résoudre $\max \sum_{i \in V} c_{i} x_{i}+\sum_{(i, j) \in E} c_{i j} y_{i j}$ s.c. $(x, y) \in P$;

2. Retirer de $P$ les contraintes non actives (sauf celles de $P_{0}$ );

3. Ajouter à $P$ les inégalités violées par la solution précédente et aller en 1 . Si aucune inégalité n'est trouvée ou si le nombre maximum d'itérations est atteint STOP.

On a choisi pour $P_{0}$, le polyèdre défini par les inégalités suivantes :

$$
\begin{cases}\sum_{i \in V} a_{i} x_{i} \leq b & (\text { sac-à-dos }) \\ \sum_{i \in \Gamma(a)} a_{i} y_{i a} \leq\left(b-a_{a}\right) x_{a} & \forall a \in V \quad(\mathrm{C} 0) \\ 0 \leq y_{i j} \leq x_{i}, 0 \leq y_{i j} \leq x_{j} & \forall(i, j) \in E \\ 0 \leq x_{i} \leq 1 & \forall i \in V .\end{cases}
$$

On trouve dans $P_{0}$ la contrainte initiale (sac-à-dos), la contrainte $\mathrm{C} 0$ obtenue en multipliant la contrainte de sac-à-dos par une variable $x_{a}$ selon la méthode de la section 3.1. Les contraintes $y_{i j} \leq x_{i}, y_{i j} \leq x_{j}$ sont des $c u t^{S, T}(x, y) \geq 0$ avec $S$ et $T$ de cardinal 1.

Ce polyèdre est tel que si les coûts $c_{i j}$ sont positifs (ce qui est le cas des instances habituelles) alors toute solution $(x, y)$ de l'algorithme de coupes avec $x$ en $0-1$ est solution du sac-à-dos quadratique car $y_{i j}=\min \left(x_{i}, x_{j}\right)=x_{i} x_{j}$.

La recherche d'inégalités violées (étape 3 de l'algorithme de coupes) est organisée de la façon suivante :

1. recherche d'inégalités $\mathrm{C} 1$ construites à partir de l'inégalité « coupe »;

2. recherche d'inégalités $\mathrm{C} 2, \mathrm{C} 3, \mathrm{C} 4, \mathrm{C} 5$ construites à partir de couvertures;

3. recherche d'inégalités $\mathrm{C} 6, \mathrm{C} 7, \mathrm{C} 8$ construites à partir de la contrainte de sac-à-dos. 
Détaillons un peu ces différents types d'inégalités.

1. Les inégalités $\mathrm{C} 1$ sont les inégalités « coupe » liftées ou pas de la section 2 .

2. Les inégalités $\mathrm{C} 2$ sont les inégalités de type $(1, \alpha+1)$ configuration $(c f$. Sect. 3.2, Prop. 3.2.1).

3. Les inégalités C3 sont les inégalités de type $(0, \alpha+1)$ configuration $(c f$. Sect. 3.2, Prop. 3.2.2).

4. Les inégalités $\mathrm{C} 4$ sont les inégalités obtenues par multiplication d'une ( 0 , $\alpha+1$ ) configuration (C3) par une variable directe (cf. Sect. 4.1, Prop. 4.1.1).

5. Les inégalités C5 sont les inégalités obtenues par multiplication d'une (1, $\alpha+1$ ) configuration (C2) par une variable complémentée (cf. Sect. 4.2, Prop. 4.2.1).

6. Les inégalités C6 sont celles obtenues par multiplication de la contrainte de sac-à-dos par une variable complémentée selon la méthode de la section 3.1.

7. Les inégalités $\mathrm{C} 7$ sont celles obtenues par multiplication de l'inégalité C6 par une variable directe selon la méthode de la section 4.1.

8. Les inégalités C8 sont celles obtenues par multiplication de l'inégalité C0 par une variable complémentée selon la méthode de la section 4.2.

La recherche des inégalités est faite de façon exhaustive pour C6, C7, C8 et de façon heuristique pour $\mathrm{C} 1$ à $\mathrm{C} 5$ selon les algorithmes exposés ci-dessous.

\section{Inégalités $\mathrm{C} 1$.}

Pour chaque clique $C$ du graphe $G$ :

- on cherche (de façon heuristique) quelques couples $S$ et $T$ disjoints et inclus dans l'ensemble des sommets de $C$ qui minimisent $c^{S} t^{S, T}(x, y)$ où $(x, y)$ est la solution courante de l'algorithme de coupes;

- pour chaque couple trouvé, les ensembles $S, T$ sont ensuite modifiés de façon à vérifier les conditions $\mathrm{C} 1$ et $\mathrm{C} 2$ de la proposition 2.1 en éliminant les éléments les plus lourds (au sens des poids $a_{i}$ );

- les inégalités obtenues sont ensuite liftées selon les résultats de la proposition 2.3 ;

- l'inégalité la plus violée est retenue et ajoutée au polyèdre $P$.

En fait toutes les cliques du graphe ne sont pas parcourues mais un algorithme simple de type glouton en donne une liste de longueur raisonnable. La taille maximum des cliques est un paramètre de l'algorithme. On a pris 10 ou 15 .

\section{Inégalités C2, C3, C4 et C5.}

Pour chacune des 4 inégalités, nous avons à chercher un ensemble $S$ t.q. le couple $t, S$ soit une $(\beta, \alpha+1)$ configuration (avec $\beta=0$ ou 1 ). La recherche utilisée est de type glouton. On recherche des couvertures constituées d'éléments consécutifs, triés selon un ordre dépendant de l'heuristique considérée. Dans une première heuristique on cherche des couvertures minimales. Les éléments sont alors classés par ordre des poids $a_{i}$ croissants. Dans une seconde heuristique les éléments 
sont classés selon un ordre basé sur les valeurs des variables $x, y$ et dépendant de l'inégalité considérée.

Supposons les éléments triés selon l'ordre voulu.

On pose $i=1$.

On cherche $\alpha$ t.q. $a_{i}+a_{i+1}+\ldots+a_{i+\alpha-1} \leq b$ et $a_{i}+a_{i+1}+\ldots+a_{i+\alpha-1}+a_{i+\alpha}>b$. Si $\alpha$ est trouvé, alors $S=\{i, i+1, \cdots, i+\alpha\}$ est une couverture. On pose $i=i+1$ et on réitère.

Quand la liste est ordonnée par poids croissants, on obtient facilement une couverture minimale en retirant de $S$ les éléments de $i$ à $i+j-1$ où $j \geq 0$ est le plus petit entier t.q. $S-\{i, \ldots, i+j\}$ n'est pas une couverture.

Les couvertures trouvées sont toujours complétées par l'ensemble des éléments de poids supérieur ou égal au plus grand des poids de la couverture (extension de couverture).

Ce sont les inégalités les plus violées qui sont retenues et ajoutées au polyèdre $P$.

Notons enfin que le nombre d'itérations maximum de l'algorithme de coupes est 150 .

\subsection{JEUX D'ESSAIS}

Les jeux d'essais ont été générés aléatoirement de la manière suivante : les coefficients $c_{i}, c_{i j}$ non nuls sont des entiers tirés aléatoirement entre 1 et 100, les $a_{i}$ sont des entiers tirés entre 1 et 50 et $b$ est un entier tiré entre 50 et $\sum_{i \in V} a_{i}$. La fonction objectif est générée avec différentes densités $\frac{\text { nombre de coefficients non nuls }}{\frac{1}{2}|V|(|V|+1)}$ allant de 25 à $100 \%$. Les tailles des problèmes (i.e. $|V|)$ vont de 50 à 150 .

Les résultats sont rassemblés dans le tableau suivant. On appelle borne inf. soit l'optimum du problème obtenu par Branch \& Bound ou soit la valeur d'une solution approchée fournie par l'heuristique de Billionnet et Calmels [2]. Les 2 premières colonnes concernent les jeux d'essais :

1. $n$ est la taille du problème (cardinal de $V$ );

2. densité est la densité de la fonction objectif.

Les colonnes qui suivent concernent les résultats relatifs à notre algorithme de coupes;

3. $\mathrm{C} i(i=1$ à 8$)$ est le nombre de contraintes de type $\mathrm{C} i$ générées;

4. $\Delta$ init est l'écart relatif entre la borne sup. fournie à la l'itération initiale de l'algorithme de coupes et la borne inf;

5. $\Delta$ fin est l'écart relatif entre la borne sup. fournie à la fin de l'algorithme de coupes et la borne inf;

6. gain est le ratio $\frac{\text { borne sup. initiale-borne sup. finale }}{\text { borne sup. initiale-borne inf. }}$. Il mesure la contribution des coupes à la progression de la borne supérieure ;

7. tps (s.) est le temps CPU en secondes.

Dans [5] une borne basée sur une méthode de décomposition est étudiée. Cette borne est comparée à la valeur d'un programme linéaire $\overline{\mathrm{LP}}$ dont les contraintes sont les inégalités $y_{i j} \leq x_{i}, y_{i j} \leq x_{j}$, les inégalités $y_{i j} \geq x_{i}+y_{j}-1$ (que nous n'avons 
pas dans notre algorithme), les inégalités C0 et C6. Á titre de comparaison, dans les dernières colonnes du tableau nous avons reporté les résultats obtenus avec le programme linéaire $\overline{\mathrm{LP}}$ :

$-\Delta \mathrm{LP}$ est l'écart relatif entre la valeur optimale de $\overline{\mathrm{LP}}$ et la borne inf;

- tps $\overline{\mathrm{LP}}(\mathrm{s}$.) est le temps CPU en secondes nécessaire à la résolution de $\overline{\mathrm{LP}}$.

Chaque ligne est une moyenne de cinq jeux d'essais. Les jeux d'essais ont été exécutés sur un Digital Alpha Serveur 800 (processeur Alpha 330 MHz). Le logiciel de programmation linéaire utilisé est CPLEX V.6.

Tableau 1. Résultats expérimentaux.

\begin{tabular}{rr|rrrrrrrrrrrr|rr}
\hline$n$ & densité & C1 & C2 & C3 & C4 & C5 & C6 & C7 & C8 & $\Delta$ init & $\Delta$ fin & gain & tps (s) & $\Delta$ LP & tps LP (s.) \\
\hline 50 & $25 \%$ & 1 & 5 & 2 & 0 & 1 & 4 & 14 & 1 & $4,63 \%$ & $4,34 \%$ & $6 \%$ & 2 & $3,00 \%$ & \\
50 & $50 \%$ & 21 & 11 & 1 & 25 & 5 & 4 & 172 & 26 & $5,04 \%$ & $4,43 \%$ & $10 \%$ & 34 & $4,08 \%$ & 19 \\
50 & $75 \%$ & 21 & 19 & 7 & 15 & 16 & 8 & 128 & 20 & $1,74 \%$ & $1,52 \%$ & $13 \%$ & 58 & $1,08 \%$ & 22 \\
50 & $100 \%$ & 143 & 54 & 104 & 122 & 63 & 34 & 39 & 35 & $1,97 \%$ & $0,76 \%$ & $64 \%$ & 242 & $1,82 \%$ & 25 \\
\hline 100 & $25 \%$ & 3 & 22 & 0 & 6 & 3 & 1 & 136 & 0 & $6,28 \%$ & $5,88 \%$ & $6 \%$ & 78 & $4,50 \%$ & 18 \\
100 & $50 \%$ & 24 & 37 & 5 & 104 & 5 & 8 & 267 & 5 & $4,77 \%$ & $4,08 \%$ & $12 \%$ & 387 & $4,07 \%$ & 339 \\
100 & $75 \%$ & 277 & 38 & 15 & 96 & 45 & 12 & 261 & 36 & $2,57 \%$ & $2,23 \%$ & $13 \%$ & 1102 & $1,16 \%$ & 505 \\
100 & $100 \%$ & 119 & 63 & 190 & 206 & 105 & 35 & 5 & 15 & $0,85 \%$ & $0,35 \%$ & $51 \%$ & 1686 & $0,57 \%$ & 618 \\
\hline 150 & $25 \%$ & 15 & 22 & 3 & 28 & 3 & 2 & 287 & 0 & $6,75 \%$ & $6,34 \%$ & $6 \%$ & 279 & $6,58 \%$ & 388 \\
150 & $50 \%$ & 38 & 33 & 8 & 26 & 20 & 6 & 233 & 1 & $3,43 \%$ & $3,00 \%$ & $9 \%$ & 705 & $2,80 \%$ & 2192 \\
150 & $75 \%$ & 99 & 53 & 7 & 173 & 16 & 7 & 419 & 1 & $1,80 \%$ & $1,47 \%$ & $17 \%$ & 3653 & $0,36 \%$ & 4344 \\
150 & $100 \%$ & 144 & 59 & 268 & 284 & 332 & 49 & 5 & 29 & $0,43 \%$ & $0,33 \%$ & $32 \%$ & 6921 & $0,38 \%$ & 4602 \\
\hline
\end{tabular}

\subsection{Commentaires}

Comparé à l'écart relatif $\Delta \overline{\mathrm{LP}}$, on voit que $\Delta$ fin est moins bon pour les densités $\leq 75 \%$. La raison en est que $\overline{\mathrm{LP}}$ est formulé avec toutes les variables $y_{i j}$ c'est-à-dire y compris celles associées à des coefficients $c_{i j}$ nuls. La projection de LP sur les variables $y$ associées aux coefficients non nuls n'est semble-t-il pas entièrement décrite par les inégalités que nous utilisons dans notre algorithme de coupes. Cependant cette perte de précision est compensée par un gain en temps CPU important. Le temps de calcul de $\overline{\mathrm{LP}}$ est à peu près constant (ou tout au moins du même ordre de grandeur) quelle que soit la densité alors que dans notre formulation le temps de calcul diminue de façon significative avec la densité (cf. colonnes tps, Tab. 1).

Partant d'un écart relatif initial $\Delta$ init relativement bas (surtout pour les fortes densités), il s'avère difficile de l'améliorer c'est-à-dire d'atteindre un gain élevé. L'observation des résultats montre l'importance des inégalités à base de couvertures (C2-C5) pour obtenir un bon gain. En effet on remarque que le gain progresse avec la densité avec une valeur faible pour les densités $\leq 75 \%$ et relativement élevée pour la densité $100 \%$. Par ailleurs les graphiques qui suivent, montrent la corrélation entre cette augmentation et l'accroissement du nombre de contraintes à base de couvertures générées. Le faible nombre de contraintes à base de couvertures générées pour les densités $\leq 75 \%$ s'explique par la difficulté à trouver des couvertures. En effet les couvertures sont cherchées dans le voisinage d'un sommet 
(cf. Sect. 3), or le nombre moyen de voisins d'un sommet baisse avec la densité alors que le cardinal moyen d'une couverture, ne dépendant que de la contrainte de sac-à-dos, lui reste constant.

On note aussi sur les graphiques une certaine complémentarité entre les inégalités construites à partir de la contrainte de sac-à-dos $(\mathrm{C} 6-\mathrm{C} 8)$ et les inégalités à base de couvertures $(\mathrm{C} 2-\mathrm{C} 5)$ dans le sens où lorsque les premières sont en nombre, les secondes ne le sont pas et réciproquement. Cependant les inégalités basées sur la contrainte de sac-à-dos semblent avoir moins d'influence sur le gain.

Pour conclure l'analyse de ces résultats, nous voyons qu'il serait nécessaire pour l'implémentation d'un algorithme de type Branch \& Cut de concentrer l'effort sur l'amélioration des heuristiques de recherche de couvertures surtout pour les densités $\leq 75 \%$.

\section{influence des contraintes sur le gain - $\mathrm{n}=50$}

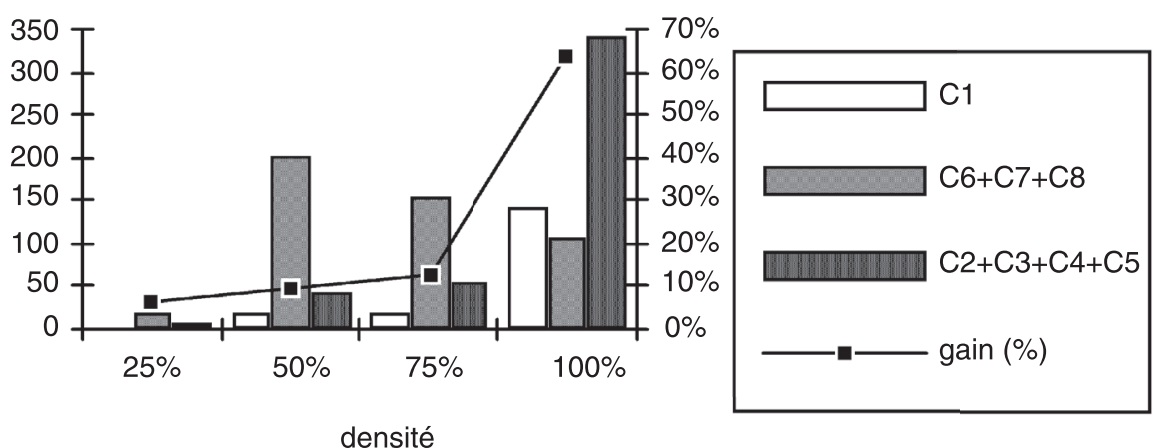

\section{Conclusion}

Nous avons construit des facettes du polytope du sac-à-dos quadratique selon deux approches différentes, l'une consistant à travailler sur l'inégalité « coupe »

\section{influence des contraintes sur le gain $-\mathrm{n}=100$}

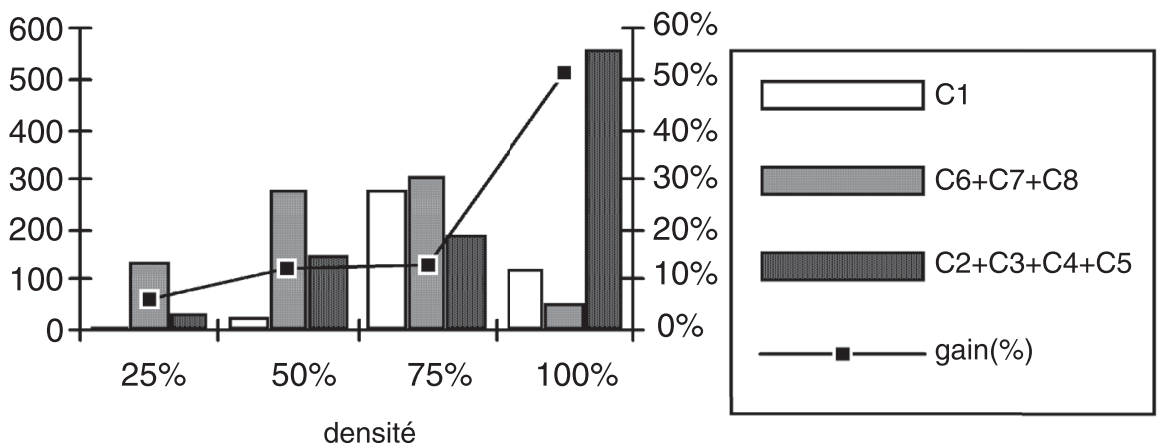


influence des contraintes sur le gain - $\mathrm{n}=150$

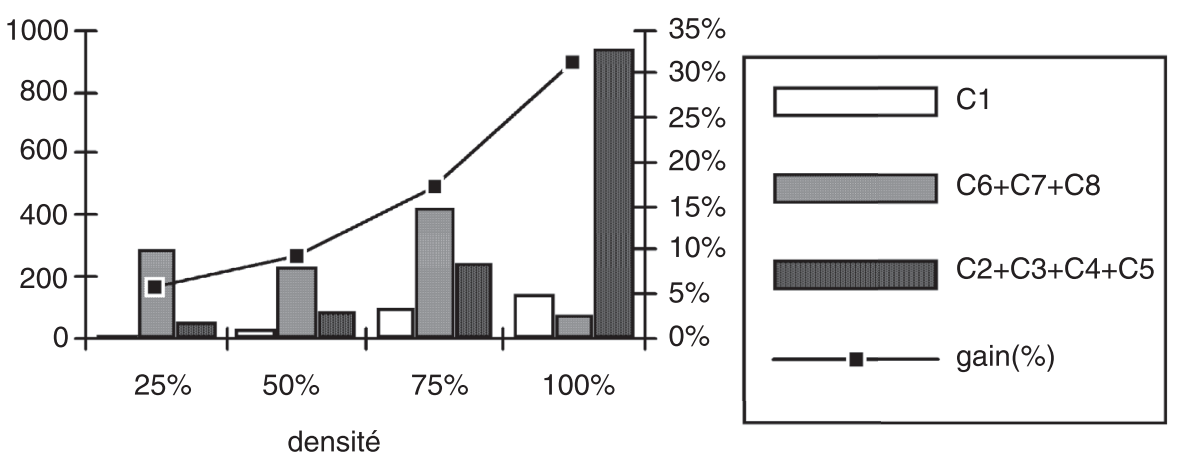

introduite par Padberg [12] pour le Boolean quadric polytope et l'autre consistant en la multiplication d'inégalités par une variable directe ou complémentée selon une approche inspirée de la méthode de linéarisation d'Adams et Sherali [1].

Pour la première approche, nous avons donné les conditions nécessaires et suffisantes pour que l'inégalité «coupe », induisant une facette du Boolean quadric polytope, induise également une facette du polytope du sac-à-dos quadratique. De plus, lorsque ces conditions ne sont pas remplies, nous avons donné un lifting particulier qui permet d'en faire une facette. Nous avons vu qu'il en existe d'autres et il serait sans doute intéressant de prolonger l'étude dans ce sens.

Il serait aussi intéressant d'étendre l'étude à d'autres inégalités valides pour le Boolean quadric polytope comme, par exemple, à l'inégalité "clique".

En ce qui concerne la deuxième approche, nous avons montré comment, à partir d'inégalités valides pour le sac-à-dos linéaire et à travers l'application en chaine des sections 3 et 4 , construire de nouvelles inégalités valides induisant des facettes du polytope du sac-à-dos quadratique.

Cette méthodologie a été appliquée à l'inégalité de couverture mais pourrait bien sûr être appliquée à d'autres inégalités valides pour le sac-à-dos linéaire et en particulier elle pourrait être appliquée aux liftings de l'inégalité de couverture.

L'intérêt de cette approche est sa simplicité et la garantie d'obtenir des facettes sous des conditions raisonnables.

Enfin, notons qu'il serait intéressant de généraliser les résultats des sections 3 et 4 à des programmes quadratique en $0-1$ soumis à plusieurs, au lieu d'une, contraintes linéaires.

D'un point de vue numérique, les résultats expérimentaux ont montré l'importance des inégalités construites à partir de couvertures pour obtenir une bonne relaxation continue du problème. Il serait intéressant d'améliorer ou d'utiliser d'autres heuristiques de recherche de couvertures surtout pour les densités de la fonction objectif faibles et moyennes. 


\section{RÉFÉRENCES}

[1] W.P. Adams et H.D. Sherali, A tight linearization and an algorithm for zero-one quadratic programming problem. Manage. Sci. 32 (1986) 1274-1289.

[2] A. Billionnet et F. Calmels, Linear programming for the 0-1 quadratic knapsack problem. Eur. J. Oper. Res. 92 (1996) 310-325.

[3] A. Billionnet, A. Faye et E. Soutif, A new upper bound for the 0-1 quadratic knapsack problem. Eur. J. Oper. Res. 112 (1999) 664-672.

[4] P. Chaillou, P. Hansen et Y. Mahieu, Best network flow bounds for the quadratic knapsack problem. Lect. Notes Math. 1403 (1986) 226-235.

[5] S. Elloumi, A. Faye et E. Soutif, Decomposition and linearization for 0-1 quadratic programming. Ann. Oper. Res. 99 (2000) 79-93.

[6] L.F. Escudero, A. Garin et G. Pérez, An O(nlogn) procedure for identifying facets of the knapsack polytope. Oper. Res. Lett. 31 (2003) 211-218.

[7] C. Helmberg, F. Rendl et R. Weismantel, A semidefinite programming approach to the quadratic knapsack problem. J. Comb. Optim. 4 (2000) 197-215.

[8] E.J. Johnson, A. Mehrotra et G.L. Nemhauser, Min-cut clustering. Math. Program. 62 (1993) 133-152.

[9] A. Mehrotra, Cardinality constrained Boolean quadratic polytope. Discrete Appl. Math. 79 (1997) 137-154.

[10] P. Michelon et L. Veilleux, Lagrangean methods for the 0-1 quadratic knapsack problem. Eur. J. Oper. Res. 92 (1996) 326-341.

[11] G.L Nemhauser et L.A. Wolsey, Integer and Combinatorial Optimization. Wiley Intersci. Ser. Discrete Math. Optim. (1988).

[12] M. Padberg, The boolean quadric polytope : some characteristics, facets and relatives. Math. Program. 45 (1989) 139-172.

[13] D.J. Rader, Valid inequalities and facets of the quadratic 0-1 knapsack polytope. Rutcor Research Report 16-97 (1997) 11 p.

[14] D.J. Rader, Lifting results for the quadratic 0-1 knapsack polytope. Rutcor Research Report 17-97 (1997) $27 \mathrm{p}$.

[15] M.G.C. Resende, K.G. Ramakrishnan et Z. Drezner, Computing Lower Bounds for the Quadratic assignment problem with an interior point algorithm for linear programming. Oper. Res. 43 (1995) 781-791.

[16] E. Soutif, Résolution du problème de sac-à-dos quadratique en variables bivalentes. Thèse de doctorat du CNAM Paris (2000).

[17] E. Zemel, Lifting the facets of zero-one polytopes. Math. Program. 15 (1978) 268-277. 